\title{
Thalamic Contributions to Basal Ganglia-Related Behavioral Switching and Reinforcement
}

\author{
Yoland Smith, ${ }^{1,2}$ D. James Surmeier, ${ }^{3}$ Peter Redgrave, ${ }^{4}$ and Minoru Kimura ${ }^{5}$ \\ ${ }^{1}$ Yerkes National Primate Research Center and ${ }^{2}$ Department of Neurology, Emory University, Atlanta, Georgia 30329, ${ }^{3}$ Department of Physiology, Feinberg \\ School of Medicine, Northwestern University, Chicago, Illinois 60611, ${ }^{4}$ Neuroscience Research Unit, Department of Psychology, University of Sheffield, \\ Sheffield S10 2TP, United Kingdom, and 5Brain Science Institute, Tamagawa University, Machida, Tokyo 194-8610, Japan
}

\begin{abstract}
Although the existence of prominent connections between the intralaminar thalamic nuclei and the basal ganglia has long been established, the limited knowledge of the functional relevance of this network has considerably hampered progress in our understanding of the neural mechanisms by which the thalamostriatal system integrates and regulates the basal ganglia circuitry. In this brief commentary, we will address this gap of knowledge through a discussion of the key points of a symposium entitled "Thalamic Contributions to Basal Ganglia-Related Behavioral Switching and Reinforcement” that will be presented at the 2011 Society for Neuroscience meeting. Recent anatomical and physiological data that support the role of the thalamostriatal system in action selection, attentional shifting, and reinforcement will be discussed. We will also address the possibility that degeneration of the thalamostriatal system could underlie some of the deficits in redirection of attention in response to salient stimuli seen in Parkinson's disease.
\end{abstract}

\section{Introduction}

In 1949, Moruzzi and Magoun showed that electrical stimulation of the midbrain reticular formation (MRF) awakens and arouses animals (Moruzzi and Magoun, 1949), while lesion of this region elicits a comatose state, and abolishes arousal responses to sensory stimuli (Lindsley et al., 1950; French et al., 1952). This major discovery was followed by electrophysiological and anatomical evidence that the MRF mediates these powerful effects upon brain activation via the so-called "ascending reticular activating system," which flows through the intralaminar nuclei, a nuclear group associated with the internal medullary lamina of the thalamus commonly seen as the source of the "nonspecific" thalamocortical system (Steriade and Glenn, 1982; Jones, 2007). Toward the posterior end of this nuclear group sits the caudal intralaminar nuclear complex, which, in primates, comprises the center median and parafascicular nuclei (CM/Pf). Almost 50 years after the publication of Magoun's reports came the study of Kinomura and colleagues showing selective increase in regional cerebral blood flow in the MRF and CM/Pf of human subjects as they go from a relaxed awake state to an attention-demanding reactiontime task, thereby providing a direct evidence that these brain areas might control the transition from relaxed wakefulness to a state of vigilance and general attention (Kinomura et al., 1996).

In this brief commentary, we will discuss recent evidence that the $\mathrm{CM} / \mathrm{Pf}$ is a key element of the ascending reticular activating system that is very sensitive to salient attention-related stimuli,

Received Sept. 11, 2011; revised Sept. 20, 2011; accepted Sept. 21, 2011.

The funding support for the work presented in this review was provided by the National Institutes of Health (Y.S., D.J.S.), the Ministry of Education, Culture, Sports, Science, and Technology of Japan (M.K.), and the Wellcome Trust (P.R.).

Correspondence should be addressed to Dr. Yoland Smith, Yerkes National Primate Research Center, Emory University, 954 Gatewood Road NE, Atlanta, GA 30329. E-mail: ysmit01@emory.edu.

DOI:10.1523/JNEUROSCI.4634-11.2011

Copyright $\odot 2011$ the authors $\quad 0270-6474 / 11 / 3116102-05 \$ 15.00 / 0$ and that the strong relationships between the CM/Pf and the striatum provides a substrate through which these sensory events may contribute to behavioral switching and reinforcement. We will also discuss the possibility that the degeneration of the thalamostriatal system may underlie some of the setshifting impairments seen in Parkinson's disease. This review will not attempt at covering the extensive literature related to the thalamostriatal system, but rather highlight recent key anatomical and physiological elements that support the role of this neural network in attentional behavior. More extensive reviews of the literature about the thalamostriatal systems have been published (Groenewegen and Berendse, 1994; Kimura et al., 2004; Smith et al., 2004, 2009, 2010; McHaffie et al., 2005; Minamimoto et al., 2009).

\section{Origin and anatomical organization of the thalamostriatal systems}

The thalamus, including the intralaminar nuclei, is an evolutionary ancient part of the vertebrate brain that predates the expansion of the cerebral cortex (Butler, 1994). Although the intralaminar nuclei are commonly seen as the sources of the "nonspecific" thalamocortical system, it has become clear over the years that the main target of intralaminar nuclei projections, most particularly those from the $\mathrm{CM} / \mathrm{Pf}$, is the striatum (Groenewegen and Berendse, 1994; Smith et al., 2004, 2009, 2010), which, like the thalamus, is a correspondingly ancient component of the vertebrate brain (Reiner, 2010, Stephenson-Jones et al., 2011). Based on its preferential targeting of segregated striatal regions, the primate CM/Pf gains access to all functional territories of the striatum: the rostral third of Pf is the main source of inputs to the limbic ventral striatum (i.e., nucleus accumbens) and the caudal tier of Pf is preferentially connected with associative striatal regions (i.e., caudate nucleus and precommissural putamen), whereas the $\mathrm{CM}$ is a major source of inputs to the sensorimotor striatum (i.e., postcommis- 
sural putamen). In rodents, the lateral part of Pf is considered as the homolog of the primate $\mathrm{CM}$, whereas the medial Pf displays strong similarities with the Pf proper (Groenewegen and Berendse, 1994; Smith et al., 2004, 2009). Overall, the organization of striatal projections from the different parts of the rat Pf is consistent with the organization of the $\mathrm{CM} / \mathrm{Pf}$-striatal system in primates. In turn, the $\mathrm{CM} / \mathrm{Pf}$ is a major target of basal ganglia outflow from the internal globus pallidus and the substantia nigra pars reticulata (Sidibé et al., 2002). Through these reciprocal connections between the basal ganglia and $\mathrm{CM} / \mathrm{Pf}$, subcortical basal ganglia-thalamostriatal loops that process limbic, associative, and sensorimotor information have been proposed (Smith et al., 2004, 2009, 2010).

Although the CM/Pf represents the main source of thalamic inputs to the striatum, the thalamostriatal system also originates from other thalamic nuclei. In contrast to projections from the $\mathrm{CM} / \mathrm{Pf}$, which terminate massively throughout the striatum, while providing minor inputs to the cerebral cortex, the rostral intralaminar, associative, and relay thalamic nuclei send major inputs to specific cortical areas, while contributing modest to sparse innervation of functionally related areas of the dorsal and ventral striatum (Smith et al., 2004, 2009, 2010). At the synaptic level, $>70-80 \%$ of CM/Pf projections target dendritic shafts of striatal projection neurons and interneurons, most particularly cholinergic interneurons, whereas $>90 \%$ of synaptic inputs from other thalamic nuclei terminate upon spines of striatal projection neurons (Sidibé and Smith, 1999; Raju et al., 2006). In light of these striking anatomical differences, the thalamostriatal system is considered as a complex network with a dual origin: the $\mathrm{CM} / \mathrm{Pf}$ or other thalamic nuclei (Smith et al., 2009, 2010).

\section{The thalamostriatal systems: a path for attention-related stimuli to the basal ganglia circuitry}

Despite compelling evidence of robust connections between the intralaminar nuclei and the striatum, the lack of functional data about the thalamostriatal system has hampered considerably our understanding of the functional organization of this neural network and its integration within the basal ganglia circuitry. On the other hand, recent cumulating evidence suggests that the transmission of salient sensory stimuli through the thalamostriatal systems plays an important role in attentional shifting, behavioral switching, and reinforcement processes, most likely through regulation of the corticostriatal circuitry via activation of striatal projection neurons and cholinergic interneurons (Minamimoto et al., 2005; Ding et al., 2010; Redgrave et al., 2010a; Smith et al., 2010).

\section{The CM/Pf-striatal system and complementary process to response bias}

Through a series of electrophysiological and behavioral studies, Kimura and colleagues have set the stage for significant advances in our understanding of the physiological properties of $\mathrm{CM} / \mathrm{Pf}$ neurons in awake nonhuman primates (Matsumoto et al., 2001; Minamimoto and Kimura, 2002; Kimura et al., 2004; Minamimoto et al., 2005, 2009). Single-unit recordings showed that visual, auditory, and somatosensory stimuli evoke brisk responses of both CM and Pf neurons. However, these are generated with a different latency; while Pf neurons are activated by these multimodal sensory stimuli at a short latency (so-called SLF neurons), CM neurons display a long-latency (so-called LLF neurons) response (Matsumoto et al., 2001). These responses are enhanced when the stimuli appear unexpectedly, but habituate when the stimuli come repeatedly. Because these responses are especially pronounced when they appear unexpectedly, involvement in the processing and integration of attention-related stimuli was proposed (Minamimoto and Kimura, 2002; Kimura et al., 2004).

It is noteworthy that salient events such as cues for task start and reward outcome evoke a pause response of tonically active neurons (TANs, presumably cholinergic interneurons), while reward- and no-reward-predicting stimuli activate subsets of phasically active neurons (PANs, presumably GABAergic projection neurons) in the striatum. Remarkably, the pause responses of TANs are almost completely eliminated by chemical inactivation of CM/Pf complex, suggesting involvement of the CM/Pfstriatal projection in regulating responses of TANs to salient sensory stimuli (Matsumoto et al., 2001).

Further characterization of $\mathrm{CM}$ responses to reward-related stimuli provided evidence that CM neurons may be involved in a mechanism complementary to decision and action bias (Minamimoto et al., 2005). For instance, during visually instructed button press tasks for large- and small-reward outcomes, a majority of CM-LLF neurons are activated when a small-reward action is required, while monkeys expect a large-reward option (Minamimoto et al., 2005). The activation by small-reward action request is especially pronounced under high level of preexisting response bias, estimated by short reaction time (RT) of GO response for large reward $[\mathrm{GO}(+\mathrm{R})]$ and long RT for small reward $[\mathrm{GO}(-\mathrm{R})]$. In sharp contrast, a subset of $\mathrm{CM}$ neurons are unresponsive to sensory stimuli (NS type) examined outside the behavioral task, but they are selectively activated when the expected large-reward request actually occurs (Minamimoto et al., 2005). Together, these results suggest specific involvement of CM neuron subtypes in response bias and its complementary process; i.e., LLF neurons play a major role in canceling preexisting response bias and switch to unexpected actions, while NS neurons facilitate processes of large-reward, biased actions. These functions may be most efficiently achieved through complex regulatory effects of the CM/Pf-striatal system upon cholinergic interneurons and projection neurons, as well as through thalamocortical external loops.

\section{Thalamic regulation of corticostriatal signaling: the role of cholinergic interneurons}

One of the main targets of $\mathrm{CM} / \mathrm{Pf}$-striatal projections are the cholinergic interneurons, recognized by their critical role in associative learning (Aosaki et al., 1994; Sidibé and Smith, 1999). Once activated, striatal acetylcholine can either mediate inhibitory effects toward corticostriatal signaling through activation of presynaptic M2 class muscarinic receptors on cortical glutamatergic afferents, or have the opposite effect via activation of postsynaptic M1 class muscarinic receptors, which can further enhance corticostriatal depolarizing influences in striatal projection neurons. In a recent series of studies using brain slice preparations that preserve corticostriatal and thalamostriatal connectivity, Surmeier and colleagues provided evidence that thalamic activation induces a burst-pause pattern of physiological responses in cholinergic interneurons that mimics the activity pattern recorded in vivo following presentation of salient stimuli (Matsumoto et al., 2001; Ding et al., 2010). These observations, combined with the fact that CM lesion abolishes this typical response pattern in primates, indicate that the CM/ Pf-striatal projection is a key regulator of cholinergic interneurons activity (Matsumoto et al., 2001). Ding et al. (2010) proposed the following mechanisms by which thalamic stimulation could gate corticostriatal signaling and control action sup- 
pression. In brief, thalamic stimulation induces an initial burst of activity in cholinergic interneurons, which leads to a transient release of acetylcholine. This increased cholinergic activity then induces a temporary suppression of excitatory cortical and thalamic drive upon both classes of striatal projection neurons via activation of presynaptic M2 muscarinic receptors. Simultaneously, a slower $(\sim 1 s)$ M1 muscarinic receptor-mediated postsynaptic facilitation of dendritic excitability to cortical inputs is initiated specifically in striatopallidal neurons, thereby providing a condition that favors activation of the "indirect" striatopallidal system over the "direct" striatonigral network. Because cholinergic interneurons are silent during this period, they cannot depress corticostriatal signaling, which results in an increased outflow from the indirect pathway, known for its role in action suppression, thereby transmitting a no-go signal to the motor thalamus (Wichmann and DeLong, 1996). In light of these data, Surmeier and colleagues suggested that this subcortical microcircuitry could be a key component of the physiological responses elicited in the basal ganglia by salient sensory stimuli, and the neural underpinning of attentional shifts and cessation of ongoing motor activity in response to salient environmental stimuli (Ding et al., 2010).

\section{$\mathrm{CM} / \mathrm{Pf}$ degeneration in Parkinson's disease: can this impair attentional shift and switching behavior?}

Parkinson's disease (PD) is clinically identified by the motor signs of bradykinesia, rigidity, and tremor at rest. In addition, many PD patients suffer from cognitive deficits, such as impairment in attention tasks, working memory, set shifting, and cognitive flexibility related to difficulty in planning, organizing, and regulating goal-directed behavior (Marder and Jacobs, 2008; Burn, 2011). In fact, PD patients have a decreased capacity to engage in normal automatic (habitual) control of actions, and become increasingly dependent on a goal-directed mode of action, which impedes their normal daily activities (see Redgrave et al., 2010b for a review). Although degeneration of the nigrostriatal dopaminergic system remains the key pathological feature of $\mathrm{PD}$, it is clear that many other neural systems are also affected, including the CM/Pf-striatal projection. Evidence from postmortem human brain studies demonstrates 30-40\% neuronal loss in the CM/Pf complex of PD patients (Henderson et al., $2000 a, b)$. This thalamic degeneration appears to be specific to $\mathrm{CM} / \mathrm{Pf}$ because neighboring thalamic nuclei remain intact. A similar pattern of degeneration was recently found in the $\mathrm{CM} / \mathrm{Pf}$ complex of parkinsonian monkeys chronically treated for many months with low doses of MPTP (1-methyl-4-phenyl1,2,3,6-tetrahydropyridine) (Villalba et al., 2011). Thus, it appears that the role of the CM/Pf-striatal system in mediating responses to salient environmental stimuli may be significantly disrupted in Parkinson's disease.

PD patients, indeed, display significant impairments in set shifting, a deficit usually related to prefrontal cortex-striatal dysfunction, and loss of striatal and cortical dopamine (Robbins and Arnsten, 2009). However, the CM/Pf-striatal connections could also be involved. As discussed above, the CM/Pf plays a particularly important role in redirecting attention to salient stimuli, behavioral flexibility, and changing behavior in responses to unpredicted stimuli (Matsumoto et al., 2001; Minamimoto et al., 2009). Degeneration of caudal intralaminar nuclei, with corresponding loss of modulation over the activity of TANs (i.e., cholinergic interneurons) and intrastriatal circuitry, would result in deficiencies in the ability to switch attention and reselect a proper action under changing circumstances. In this manner, the loss of
$\mathrm{CM} / \mathrm{Pf}$ neurons could be one of the contributing factors to the set-shifting inability in PD. Future studies in MPTP-treated monkeys and patients with PD are necessary to further address this issue.

\section{The superior colliculus: an integrative processing center of attention-related information to the basal ganglia-thalamostriatal loops}

Although the exact sources of salient stimuli that engage the thalamostriatal systems into complex attentional behaviors remain to be determined, Redgrave and colleagues have suggested that the superior colliculus displays the evolutionary profile, anatomical connectivity, and physiological features consistent with a major role in attentional shifting and reinforcement learning (McHaffie et al., 2005; Redgrave et al., 2010). The basal ganglia and the superior colliculus (optic tectum in primitive species) are neural structures that appeared early ( $>400$ million years ago) and have been highly conserved throughout the evolution of the vertebrate brain (Reiner, 2010; Stephenson-Jones et al., 2011), thereby suggesting that they are part of fundamental processing units that play basic functions in mammalian behavior.

In light of various anatomical studies, McHaffie et al. (2005) proposed the existence of subcortical tecto-basal ganglia loops that interconnect the superficial and deep layers of the superior colliculus with specific thalamic nuclei, which, in turn, gain access to the basal ganglia circuitry via prominent thalamostriatal connections, or connections with other basal ganglia nuclei, such as the subthalamic nucleus and the substantia nigra pars compacta (see also Redgrave et al., 2010a). The looped architecture of these connections could resolve fundamental selection issues associated with responses to biologically salient stimuli. It is clear that the natural world, indeed, contains multiple stimuli, many of which can be represented simultaneously in structures like the superior colliculus. Then, the problem is to select the proper stimulus to guide behavior and decide whether it should be approached or avoided. Recent experiments demonstrate a behavioral approachavoidance conflict in which appetitive and threatening stimuli evoked neural activation in different subregions of the superior colliculus (Redgrave et al., 1999). Subsequent anatomical experiments showed that these functionally segregated regions engage different tecto-thalamo-striatal loops projecting through the basal ganglia. Selective disinhibition imposed by the basal ganglia on return connections to the different subregions of the superior colliculus (Chevalier and Deniau, 1990) could offer a potential resolution of the observed behavioral approach-avoidance conflict (Redgrave et al., 1999).

Another important issue that remains to be addressed is the mechanism by which short-latency sensory signals in the superior colliculus may be used by the basal ganglia for a particular form of reinforcement learning. A basic problem for any autonomous agent is to identify those events in the world for which it is responsible, and then to discover what it is doing that is causal. Part of the basal ganglia's connectional architecture ensures they receive a running copy of behavioral output (Reiner et al., 2003). An unpredicted and biologically significant behavioral event causes a short-latency response in the superior colliculus, which is relayed to the basal ganglia via the intralaminar thalamostriatal projections (Dommett et al., 2005; Coizet et al., 2007; Schulz et al., 2009). These signals are thought to reinforce (increase the probability of reselecting) immediately prior behaviors (Redgrave and Gurney, 2006). If the reinforced behavior caused the event, its reselection will cause the event to reoccur. Through trial and error repetition, the system can then learn what aspect(s) of 
its behavior is producing the event. The advantage of having short-latency sensory signals from the superior colliculus initiate the process of reinforcement is that they occur before any responses elicited by the caused event itself. Delaying reinforcement beyond this point would risk having the record of behavioral output contaminated with noncausal items, thereby exacerbating the credit-assignment problem (i.e., how to direct reinforcement specifically to causal components of prior behavior) (Izhikevich, 2007). This analysis predicts that appropriately timed signals from the superior colliculus that evoke phasic thalamostriatal (glutamate) and nigrostriatal (dopamine) inputs to the striatum should reinforce prior inputs from the cerebral cortex, thereby suggesting a novel form of sensory reinforced corticostriatal plasticity. As discussed above, complex thalamic regulation of corticostriatal signaling by cholinergic interneurons has, indeed, been proposed as a potential substrate for cessation of ongoing motor activity in response to the appearance of salient environmental stimuli (Ding et al., 2010).

Insofar as the selective properties of basal ganglia circuitry assist normal collicular function, the short-latency sensorydriven activity in the superior colliculus could be used by the basal ganglia to reinforce the discovery of agency and the development of novel actions. If so, these ancient, but conserved, neural circuits may offer a tractable model for investigating general principles of action selection and reinforcement learning.

\section{Concluding remarks}

In this brief commentary, we summarized some of the key elements that support the potential role of the CM/Pf-striatal system in action selection, attentional shifting, and reinforcement. The past decade has witnessed a significant development of our understanding of the functional anatomy of the thalamostriatal systems. The tight integration of this network within the basal ganglia circuitry and subcortical loops with the superior colliculus, combined with the drive from the reticular activating system, provides a framework through which salient sensory events can be processed and impact upon cortical and subcortical processing of attention-related stimuli. The degeneration of the CM/Pf-striatal system could underlie some of the deficits in set shifting and redirection of attention in response to changes in environmental stimuli seen in Parkinson's disease. Future studies aimed at characterizing the functional role of the $\mathrm{CM} / \mathrm{Pf}-$ striatal system in chronic nonhuman primate models of Parkinson's disease that harbor significant degeneration of the CM/Pf are needed to further assess the role of the CM/Pf-striatal system in normal and pathological conditions.

\section{References}

Aosaki T, Graybiel AM, Kimura M (1994) Effect of the nigrostriatal dopamine system on acquired neural responses in the striatum of behaving monkeys. Science 265:412-415.

Burn DJ (2011) Clinical features of dementia associated with Parkinson's disease and dementia with Lewy bodies. In: Parkinson's disease: nonmotor and non-dopaminergic features (Olanow CR, Stocchi F, Lang AE, eds), pp 134-144. Oxford: Wiley-Blackwell.

Butler AB (1994) The evolution of the dorsal thalamus of jawed vertebrates, including mammals: cladistic analysis and a new hypothesis. Brain Res Rev 19:29-65.

Chevalier G, Deniau JM (1990) Disinhibition as a basic process in the expression of striatal functions. Trends Neurosci 13:277-280.

Coizet V, Overton PG, Redgrave P (2007) Collateralization of the tectonigral projection with other major output pathways of superior colliculus in the rat. J Comp Neurol 500:1034-1049.

Ding JB, Guzman JN, Peterson JD, Goldberg JA, Surmeier DJ (2010) Thalamic gating of corticostriatal signaling by cholinergic interneurons. Neuron 67:294-307.
Dommett E, Coizet V, Blaha CD, Martindale J, Lefebvre V, Walton N, Mayhew JE, Overton PG, Redgrave P (2005) How visual stimuli activate dopaminergic neurons at short latency. Science 307:1476-1479.

French JC, Von Amerongen FK, Magoun HW (1952) An activating system in brain stem of monkey. AMA Arch Neurol Psychiatry 68:577-590.

Groenewegen HJ, Berendse HW (1994) The specificity of the 'nonspecific' midline and intralaminar thalamic nuclei. Trends Neurosci 17:52-57.

Henderson JM, Carpenter K, Cartwright H, Halliday GM (2000a) Loss of thalamic intralaminar nuclei in progressive supranuclear palsy and Parkinson's disease: clinical and therapeutic implications. Brain 123: $1410-1421$

Henderson JM, Carpenter K, Cartwright H, Halliday GM (2000b) Degeneration of the centre median-parafascicular complex in Parkinson's disease. Ann Neurol 47:345-352.

Izhikevich EM (2007) Solving the distal reward problem through linkage of STDP and dopamine signaling. Cereb Cortex 17:2443-2452.

Jones EG (2007) The thalamus, Ed 2, pp 1115-1165. Cambridge, UK: Cambridge UP.

Kimura M, Minamimoto T, Matsumoto N, Hori Y (2004) Monitoring and switching of cortico-basal ganglia loop functions by the thalamo-striatal system. Neurosci Res 48:355-360.

Kinomura S, Larsson J, Gulyás B, Roland PE (1996) Activation by attention of the human reticular formation and thalamic intralaminar nuclei. Science 271:512-515.

Lindsley DB, Schreiner LH, Knowles WB, Magoun HW (1950) Behavioral and EEG changes following chronic brain stem lesions in the cat. Electroencephalogr Clin Neurophysiol 2:483-498.

Marder KS, Jacobs DM (2008) Dementia. In: Parkinson's disease-diagnosis and clinical management (Factor SA, Weiner WJ, eds), pp 147-158. Demos: New York.

Matsumoto N, Minamimoto T, Graybiel AM, Kimura M (2001) Neurons in the thalamic CM-Pf complex supply striatal neurons with information about behaviorally significant sensory events. J Neurophysiol 85:960-976.

McHaffie JG, Stanford TR, Stein BE, Coizet V, Redgrave P (2005) Subcortical loops through the basal ganglia. Trends Neurosci 28:401-407.

Minamimoto T, Kimura M (2002) Participation of the thalamic CM-Pf complex in attentional orienting. J Neurophysiol 87:3090-3101.

Minamimoto T, Hori Y, Kimura M (2005) Complementary process to response bias in the centromedian nucleus of the thalamus. Science 308:1798-1801.

Minamimoto T, Hori Y, Kimura M (2009) Roles of the thalamic CM-PF complex-basal ganglia circuit in externally driven rebias of action. Brain Res Bull 78:75-79.

Moruzzi G, Magoun HW (1949) Brain stem reticular formation and activation of the EEG. Electroencephalogr Clin Neurophysiol 1:455-473.

Raju DV, Shah DJ, Wright TM, Hall RA, Smith Y (2006) Differential synaptology of vGluT2-containing thalamostriatal afferents between the patch and matrix compartments in rats. J Comp Neurol 499:231-243.

Redgrave P, Gurney K (2006) The short-latency dopamine signal: a role in discovering novel actions? Nat Rev Neurosci 7:967-975.

Redgrave P, Prescott TJ, Gurney K (1999) The basal ganglia: a vertebrate solution to the selection problem? Neuroscience 89:1009-1023.

Redgrave P, Coizet V, Comoli E, McHaffie JG, Leriche M, Vautrelle N, Hayes LM, Overton P (2010a) Interactions between the midbrain superior colliculus and the basal ganglia. Front Neuroanat 4:132.

Redgrave P, Rodriguez M, Smith Y, Rodriguez-Oroz MC, Lehericy S, Bergman H, Agid Y, DeLong MR, Obeso JA (2010b) Goal-directed and habitual control in the basal ganglia: implications for Parkinson's disease. Nat Rev Neurosci 11:760-772.

Reiner AJ (2010) The conservative evolution of the vertebrate basal ganglia. In: Handbook of basal ganglia structure and function (Steiner H, Tseng KY, eds), pp 29-62. New York: Elsevier.

Reiner A, Jiao Y, Del Mar N, Laverghetta AV, Lei WL (2003) Differential morphology of pyramidal tract-type and intratelencephalically projecting-type corticostriatal neurons and their intrastriatal terminals in rats. J Comp Neurol 457:420-440.

Robbins TW, Arnsten AF (2009) The neuropsychopharmacology of frontoexecutive function: monoaminergic modulation. Annu Rev Neurosci 32:267-287.

Schulz JM, Redgrave P, Mehring C, Aertsen A, Clements KM, Wickens JR, Reynolds JN (2009) Short-latency activation of striatal spiny neurons via subcortical visual pathways. J Neurosci 29:6336-6347. 
Sidibé M, Smith Y (1999) Thalamic inputs to striatal interneurons in monkeys: synaptic organization and co-localization of calcium binding proteins. Neuroscience 89:1189-1208.

Sidibé M, Paré JF, Smith Y (2002) Nigral and pallidal inputs to functionally segregated thalamostriatal neurons in the centromedian/parafascicular intralaminar nuclear complex in monkey. J Comp Neurol 447:286-299.

Smith Y, Raju DV, Pare JF, Sidibe M (2004) The thalamostriatal system: a highly specific network of the basal ganglia circuitry. Trends Neurosci 27:520-527.

Smith Y, Raju D, Nanda B, Pare JF, Galvan A, Wichmann T (2009) The thalamostriatal systems: anatomical and functional organization in normal and parkinsonian states. Brain Res Bull 78:60-68.

Smith Y, Galvan A, Raju DV, Wichmann T (2010) Anatomical and func- tional organization of the thalamostriatal systems. In: Handbook of basal ganglia structure and function: a decade of progress (Steiner $\mathrm{H}$, Tseng KY, eds), pp 381-392. New York: Elsevier.

Stephenson-Jones M, Samuelsson E, Ericsson J, Robertson B, Grillner S (2011) Evolutionary conservation of the basal ganglia as a common vertebrate mechanism for action selection. Curr Biol 21:1081-1091.

Steriade M, Glenn LL (1982) Neocortical and caudate projections of intralaminar thalamic neurons and their synaptic excitation from midbrain reticular core. J Neurophysiol 48:352-371.

Villalba RM, Wichmann T, Smith Y (2011) Neuronal loss in the caudal intralaminar nuclear group, CM/Pf, in MPTP-treated parkinsonian monkeys. Soc Neurosci Abstr 37:882.09.

Wichmann T, DeLong MR (1996) Functional and pathophysiological models of the basal ganglia. Curr Opin Neurobiol 6:751-758. 